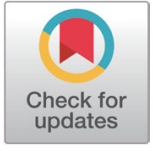

Received: May 15, 2021

Revised: Jun 18, 2021

Accepted: Jun 30, 2021

\#These authors contributed equally to this work.

*Corresponding author

Soojin Sa

Swine Science Division, National Institute of Animal Science, Rural Development Administration, Cheonan 31000 , Korea.

Tel: +82-41-580-3450

E-mail: soojinsa@korea.kr

Hakjae Chung

Swine Science Division, National Institute of Animal Science, Rural

Development Administration, Cheonan 31000 , Korea.

Tel: +82-41-580-3443

E-mail: hakjaena@korea.kr

Copyright (c) 2021 Korean Society of Animal Sciences and Technology. This is an Open Access article distributed under the terms of the Creative Commons Attribution Non-Commercial License (http:// creativecommons.org/licenses/by$\mathrm{nc} / 4.0 /$ ) which permits unrestricted non-commercial use, distribution, and reproduction in any medium, provided the original work is properly cited.

\section{Association of the ubiquitin specific peptidase 9X -linked and Afadin expression patterns with sexual maturation in boar testis}

\author{
Sun-Young Baek ${ }^{1 \#}$, Seung-Hoon Lee ${ }^{2 \#}$, Youngshin Kim", Joon-Ki Hong ${ }^{1}$, \\ Eunseok $\mathrm{Cho}^{1}$, Seungmin $\mathrm{Ha}^{3}$, Kyungwoon $\mathrm{Kim}^{4}$, Soojin $\mathrm{Sa}^{1 *}$ and \\ Hakjae Chung ${ }^{1 *}$ \\ ${ }^{1}$ Swine Science Division, National Institute of Animal Science, Rural Development Administration, \\ Cheonan 31000, Korea \\ ${ }^{2}$ Animal Biotechnology Division, National Institute of Animal Science, Rural Development Administration, \\ Wanju 55365, Korea \\ ${ }^{3}$ Dairy Science Division, National Institute of Animal Science, Rural Development Administration, \\ Cheonan 31000, Korea \\ ${ }^{4}$ Planning and Coordination Division, National Institute of Animal Science, Rural Development \\ Administration, Wanju 55365, Korea
}

\section{Abstract}

Closely correlated expression patterns between ubiquitin specific peptidase 9X-linked (USP9X) and adherens junction formation factor (Afadin) in mouse testis development suggests that Usp9x regulates the deubiquitination of Af-6 (also known as Afadin, AFDN), and subsequently, the cell adhesion dynamics during gametogenesis. However, this relationship has not yet been tested in other domestic animals. The study was examined the temporal and spatial expression patterns of porcine USP9X and AFDN from the pre-pubertal to adult stages using real time-PCR and immunohistochemistry. Furthermore, we detected the transcripts of USP9X and AFDN in the testis of 1-, 6- and 12-months old boar, respectively. USP9X and AFDN were found to have similar expressions patterns, with basal expression after 1 month followed by a significant up-regulation from 6 months (puberty) onwards. In addition, neither the AFDN or USP9X proteins were detected in spermatogenic cells but they were expressed in the leydig cells and sertoli cells. USP9X was detected around the basal lamina during pre-puberty, and predominantly expressed in the leydig cells at puberty. Finally, in adult testis, USP9X was increased at the sertoli cell-cell interface and the sertoli cell-spermatid interface. In summary, closely correlated expression patterns between USP9X and AFDN in boar testis supports the previous findings in mice. Furthermore, the junction connections between the sertoli cells may be regulated by the ubiquitination process mediated via USP9X.

Keywords: Ubiquitin specific peptidase 9X -linked (USP9X), Adherens junction formation factor (AFDN), Spermatogenesis, Spermiogenesis, Boar testis 
ORCID

Sun-Young Baek

https://orcid.org/0000-0001-5130-2269

Seung-Hoon Lee

https://orcid.org/0000-0002-4362-7538

Youngshin Kim

https://orcid.org/0000-0001-5466-8813

Joon-Ki Hong

https://orcid.org/0000-0001-8272-1263

Eunseok Cho

https://orcid.org/0000-0001-5223-099X

Seungmin $\mathrm{Ha}$

https://orcid.org/0000-0002-5152-1979

Kyungwoon Kim

https://orcid.org/0000-0003-0225-9684

Soojin Sa

https://orcid.org/0000-0002-2634-5109

Hakjae Chung

https://orcid.org/0000-0002-3127-5192

Competing interests

No potential conflict of interest relevant to

this article was reported.

Funding sources

This work was carried out with the support of "Cooperative Research Program for

Agriculture Science and Technology

Development (Project No. PJ01359301)"

Rural Development Administration, Korea.

Acknowledgements

Not applicable.

Availability of data and material Upon reasonable request, the datasets of this study can be available from the corresponding author.

Authors' contributions

Conceptualization: Chung $\mathrm{H}$.

Methodology: Baek SY, Chung H.

Software: Hong JK.

Validation: Baek SY, Chung $\mathrm{H}$.

Investigation: Baek SY, Chung H.

Gene analysis: Lee SH, Kim K.

Experimental animal management: Kim Y, Sa S.

Experimental slaughter (anesthesia): Cho E, $\mathrm{HaS}$.

Writing - review \& editing: Chung $\mathrm{H}$.

Ethics approval and consent to participate The National Institute of Animal Science Animal Care and Use Committee approved all animal procedures (No.2019-397).

\section{INTRODUCTION}

The ubiquitin-mediated degradation of certain regulatory proteins is critical for a large range of biological process, including the cell-cycle, transcriptional regulation, signal transduction, endocytosis and tumor suppression [1,2]. Ubiquitin-ligase and deubiquitinase related genes are reportedly expressed during testes development in a stage-specific manner and they are involved in spermatogenesis and spermiogenesis $[3,4]$.

Specifically, USP9X (ubiquitin specific peptidase 9x-linked) is predominantly expressed in germ cells and supporting somatic cells (Sertoli cells) in a stage-and sex-dependent manner during mouse gonadal development [5]. Moreover, a loss of function study demonstrated that germ cell-specific conditional deletion of USP9X did not affect the development of the testis during the meiotic phase, but caused apoptosis during the spermatocyte stage, aberrant spermigogenesis, leading to infertility in mice [6].

In addition, Afadin is a cytoskeletal and junction-associated protein that has been identified as a substrate for USP9X [7]. In mouse testes, Afadin is expressed in supporting cells but no germ cells throughout the postnatal and adult stages and is enriched at the sites of Sertoli-spermatid junctions and Sertoli-sertoli junction. This suggests that USP9X may affect the proteasomedependent degradation of Afadin mediated as a substrate-specific regulator in spermatogenesis and spermiogenesis in mammalian $[5,8]$. However, the expression patterns of adherens junction formation factor (AFDN) and USP9X during testis development has not yet been investigated in other domestic mammals.

In this study, we first compared amino acid sequences of pig AFDN and USP9X with those of other mammals including mouse, human, and cattle to determine whether the two genes are conserved across species. We then examined their spatiotemporal expression using quantitative real time polymerase chain reaction (qRT-PCR) and immunofluorescence using the testes from boars that were in pre-pubertal, pubertal, and adult stages of development.

\section{MATERIALS AND METHODS}

All reagents, unless stated otherwise, were purchased from Sigma-Aldrich (St. Louis, MO, USA). The National Institute of Animal Science Animal Care and Use Committee approved all animal procedures (No.2019-397). Testis tissue samples were obtained from 1, 6, and 12-month-old boar (Duroc; ten boar/growth stage) at the National Institute of Animal Science and Swine Science Division using standard castration protocols [9].

\section{Samples}

Testis tissue samples were obtained from at three boars of each age using standard castration procedures, and they were immediately placed in Hanks balanced salt solution (HBSS; Gibco, Thermo Fisher Scientific, Bohemia, NY, USA) on ice. Testes samples were preserved in Trizol for RNA isolation and in 10\% paraformaldehyde (PFA) for preservation for histological analysis of the testes tissue. The tissues were left in PFA for $24 \mathrm{~h}$ at $4{ }^{\circ} \mathrm{C}$, followed by dehydration and storage in $70 \%$ ethanol.

\section{Quantitative real-time polymerase chain reaction analysis}

Total RNA was isolated from the testes of 1 month-old (pre-pubertal), 6-month-old (pubertal) and 12-month-old (adult) pigs. According to the manufacturer's guidelines, RNA was isolated with TRIzol reagent using the RNeasy mini kit (Qiagen, Valencia, CA, USA), genomic DNA 
was removed by DNase digestion using the RNAse-free DNase kit (Qiagen), and cDNA were synthesized using SuperScript II reverse transcriptase (Invitrogen, Carlsbad, CA, USA). Real-timePCR was carried out on a StepOne Plus Real-Time PCR System (Applied Biosystems, Foster, CA, USA) using gene-specific primers as follows: USP9X forward 5'-TTG CTG TGA AGC AAT GGA AG-3' and reverse 5'- ATG CAA CAT CTG GTG CAC AT -3'; AFDN forward 5'- CAT CCA GGA CCA CGT TCT TT -3' and reverse 5'- AGG ACA CCC TGT CAC TGT CC -3'; and GAPDH forward 5'-TGA AGG TCG GAG TCA ACG GAT TTG GT-3' and reverse 5'CAT GTG GGC CAT GAG GTC CAC CAC-3'. GAPDH was used as an endogenous control to determine the relative expression levels of the target genes using the delta-delta method.

\section{Immunofluorescence}

As described previously [5], 1-, 6-, and 12-month-old boar testes were fixed with 4\% PFA, embedded in paraffin, and they were then horizontally sectioned (5- $\mu \mathrm{m}$ interval). The horizontal section samples were subjected to antigen retrieval and blocked with phosphate-buffered saline, containing $1 \%$ bovine serum albumin (BSA) and $0.2 \%$ Tween 20 (PBST) before staining. The horizontal sections were incubated with primary antibodies over night at $4{ }^{\circ} \mathrm{C}$ diluted in PBST containing $0.01 \%$ BSA, goat polyclonal IgG against the anti-USP9X (1:100, Santa Cruz Biotechnology, Santa Cruz, CA, USA), and goat polyclonal IgG against the anti-AFDN (1:100, Santa Cruz Biotechnology). Secondary antibodies were incubated for $120 \mathrm{~min}$ at room temperature, diluted in PBST containing $0.01 \%$ BSA, goat anti-rabbit IgG conjugated with fluorescein isothiocyanate (FITC).

\section{Statistical analyses}

Statistical analyses were performed by using GraphPad Prism (version 5.03, GraphPad Software, San Diego, CA, USA). Expression data were analyzed using analysis of variance (ANOVA) and presented as mean \pm standard error. $\mathrm{A} p<0.05$ was considered significant unless otherwise stated.

\section{RESULTS}

\section{Adherens junction formation factor and ubiquitin specific peptidase $9 x$-linked are} highly conserved across mammals

We performed amino acid sequence analyses using the NCBI database (HomoloGene, Constraintbased multiple alignment tool, conserved domains) and compared the sequences of pig AFDN and USP9X with those of mouse, human, and cattle. The similarity of the amino acids was found to be $81 \%$ between pig and the others, but the amino acid sequences of the functional domains were identical (Ras-associating [RA], PSD-95/Dlg/ZO-10 [PDZ] or nearly identicalForkhead association [FHA]). In pig USP9X, we found two functional domain peptidase C19C (ubiquitinyl hydrolases), and ubiquitin like fold superfamily. The overall identity of the amino acid sequences for the pig USP9X were found to be 98\%, 97\%, and 99\% for mouse, human, and cattle, respectively (Figs.1A and B).

Expression levels of adherens junction formation factor and ubiquitin specific peptidase 9x-linked are up-regulated after puberty

To determine whether the boar AFDN and USP9X genes are expressed in spermatogenic cells or somatic cells such as male germ cells in the testis, we performed qRT-PCR using boar testis obtained at pre-pubertal (1-month), pubertal (6-moths), and adult (12 months) stages of growth. Transcripts of both AFDN and USP9X were detected at the pre-pubertal stage, and regulated 
A
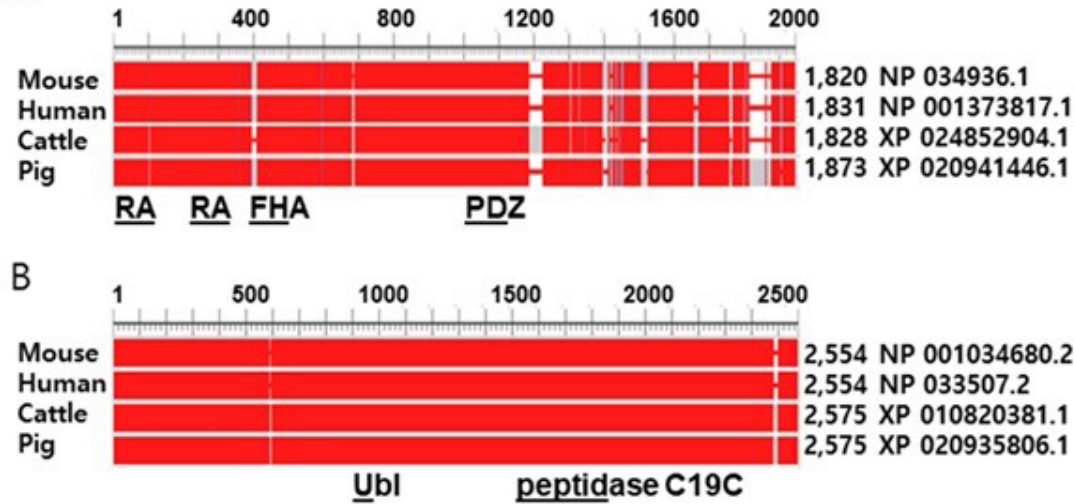

C

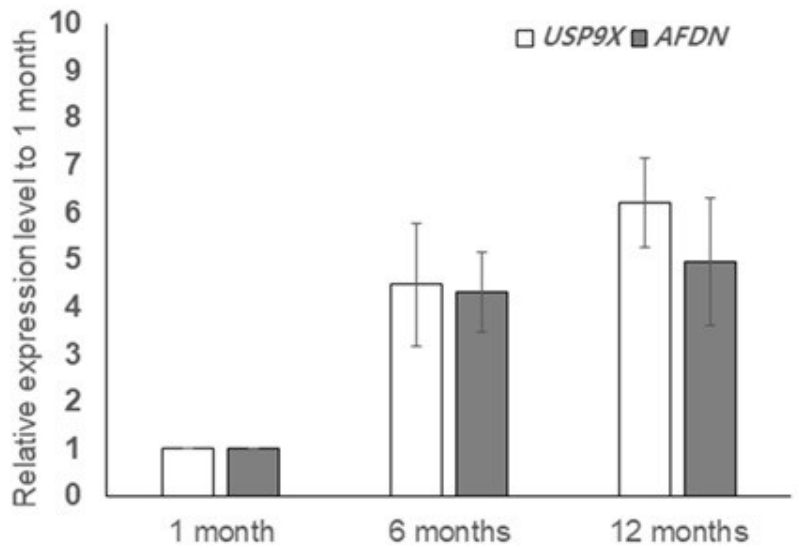

Fig. 1. Analysis of the amino acid sequences and the temporal gene expression of AFDN and USP9X. (A) Similarity of the amino acid sequences for AFDN. (B) Similarity of the amino acid sequences for USP9X. Numbers (amino acid sequences); underlines (position of domain); RA domain, FHA domain, PDZ domain, Ubl (Beta-grasp), peptidase C19C (a subfamily of peptidase C19). (C) Relative expression of AFDN and USP9X transcripts in 1-, 6-, and 12-month-old testis. RA, Ras-associated; FHA, forkhead-associated; PDZ, Postsynaptic density-95/disc large/zonula occludens; Ubl, Beta-grasp ubiquitin-like; USP9X, ubiquitin specific peptidase 9x-linked; AFDN, adherens junction formation factor.

from puberty onwards, but the expression levels were not different between the 6-month-old and 12 -month-old testis (Fig. 1C). Interestingly, we observed a tendency towards increased USP9X expression in the 12-month-old testis although the level was not significant (Fig. 1C).

Adherens junction formation factor and ubiquitin specific peptidase $9 x$-linked proteins are localized in male germ cells

To determine the localization and potential interactions between AFDN and USP9X during spermatogenesis and testis development, we examined the expression of AFDN and USP9X using immunohistochemistry. Both the AFDN and USP9X were weakly detected in interstitial cells such as the leydig cells in the 1-month-old testis, but not expressed in the seminiferous tubules (Fig. 2). AFDN proteins were detected in the sertoli cells from 6 months of age onwards, but not in any spermatogenic cells during testis development. Similarly, USP9X proteins were highly expressed in seminiferous tubule cells and the leydig cells of the adult testis. Furthermore, USP9X was detected in the basal compartment and sertoli-spermatid junctions of the seminiferous tubules in the 


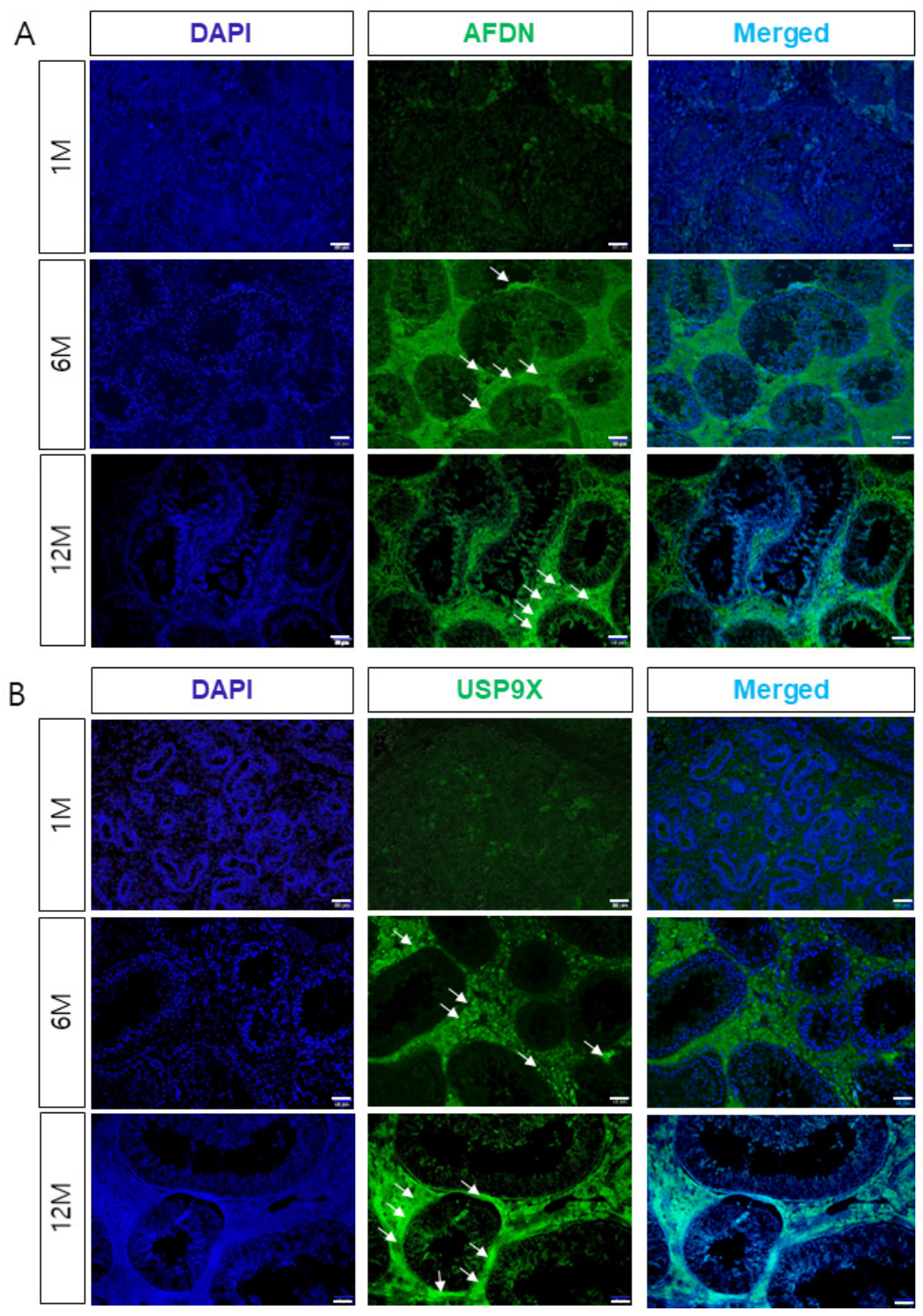

Fig. 2. Intercellular localization of AFDN and USP9X in testis. Boar AFDN (A) and USP9X (B) were detected in leydig and sertoli cells, and strongly expressed from puberty (6 months) at the sertoli-sertoli and sertolispermatid interfaces at adult (12 month). Scale bar $=50 \mu \mathrm{m}$. DAPI, 4',6-deamidino-2-phenylindole; AFDN, adherens junction formation factor, afadin; USP9X, ubiquitin specific peptidase 9X-linked.

6-month-old testes and strongly expressed at junctional sites in the 12-month-old testes.

\section{DISCUSSION}

It has been well documented that the Drosophila fat facet (faf) gene, a ubiquitin-specific protease, is required for germline development and eye formation [10,11]. Two faf homologous genes, $\mathrm{X}$-linked USP9X and Y-linked ubiquitin specific peptidase 9 (Usp9y) have previously been identified in humans and mice [12-14]. Biochemical studies have reported interactions between USP9X and the cell adhesion and signaling molecules such as afadin and $\beta$-catenin in mammalian cells, and USP9X and AFDN biologically interact and co-localize at tight junctions in polarized MDCK II 
cells $[7,15]$.

Interestingly, USP9X and AFDN, a substrate for USP9X are co-expressed at Sertoli cells and Sertoli-spermatid junctions at stages XI to VI when spermiation occurs, suggesting that the posttranslational regulation of AFDN by USP9X may control the intercellular adhesion dynamics in spermiogenesis [5]. Moreover, a loss of study showed that deuiquitinated USP9X is important during the transition from the mitotic to meiotic phase, and can result in male infertility [6].

In the present study, we first examined similarity of amino acid sequences and functional domains to determine whether AFDN and USP9X are conserved across mammals. The overall identities of the amino acid sequences of AFDN and USP9X were $>80 \%$ and $97 \%$, and the functional domains that presumably interact between AFDN and USP9X were found to be identical or nearly identical, suggesting that the expression patterns and biological functions of AFDN and USP9X in the pigs may be similar to those observed in mice $[5,6]$.

To investigate the stage-dependent intercellular expression of AFDN and USP9X during boar testes development, we collected testis samples at 1,6, and 12-months of age; testicular morphology and sertoli cell differentiation studies reported that the onset of puberty occurs at 2 to 6 months $[16,17]$. In the present study, the diameter of the seminiferous tubes was relatively small in the 1-month-old testis, but in the 6-month-old and 12-month-old testes it showed more advanced development. In addition, qRT-PCR and immunofluorescence results support developmental stage-specific expression patterns of AFDN and USP9X during testis development. Furthermore, intercellular co-localization of AFDN and USP9X in the basal compartment of the seminiferous epithelia and the seroli cells-spermatid junctions implied that USP9X may regulate the junctional dynamics of sertoli-cells via AFDN. However, the biological functions of AFDN and USP9X have still not been investigated in leydig cells during testis development, although they are strongly and continuously expressed from puberty onwards.

Taken together, close spatiotemporal expression patterns of AFDN and USP9X in boar testes that produce similar expression patterns to those previously observed in mouse testis suggests that AFDN and USP9X may have conserved biological functions during mammalian spermatogenesis, including spermiation.

\section{REFERENCES}

1. Hershko A, Ciechanover A. The ubiquitin system. Annu Rev Biochem. 1998;67:425-79. https://doi.org/10.1146/annurev.biochem.67.1.425

2. Hochstrasser M. Ubiquitin, proteasomes, and the regulation of intracellular protein degradation. Curr Opin Cell Biol. 1995;7:215-23. https://doi.org/10.1016/0955-0674(95)80031-X

3. Bose R, Manku G, Culty M, Wing SS. Ubiquitin-proteasome system in spermatogenesis. Adv Exp Med Biol. 2014;759:181-213. https://doi.org/10.1007/978-1-4939-0817-2_9

4. Suresh B, Lee J, Hong SH, Kim KS, Ramakrishna S. The role of deubiquitinating enzymes in spermatogenesis. Cell Mol Life Sci. 2015;72:4711-20. https://doi.org/10.1007/s00018-0152030-z

5. Sato T, Kanai Y, Noma T, Kanai-Azuma M, Taya S, Matsui T, et al. A close correlation in the expression patterns of Af- 6 and Usp9x in Sertoli and granulosa cells of mouse testis and ovary. Reproduction. 2004;128:583-94. https://doi.org/10.1530/rep.1.00060

6. Kishi K, Uchida A, Takase HM, Suzuki H, Kurohmaru M, Tsunekawa N, et al. Spermatogonial deubiquitinase USP9X is essential for proper spermatogenesis in mice. Reproduction. 2017;154:135-43. https://doi.org/10.1530/REP-17-0184

7. Taya S, Yamamoto T, Kano K, Kawano Y, Iwamatsu A, Tsuchiya T, et al. The Ras target AF-6 
is a substrate of the Fam deubiquitinating enzyme. J Cell Biol. 1998;142:1053-62. https://doi. org/10.1083/jcb.142.4.1053

8. Cheng CY, Mruk DD. Cell junction dynamics in the testis: sertoli-germ cell interactions and male contraceptive development. Physiol Rev. 2002;82:825-74. https://doi.org/10.1152/physrev.00009.2002

9. Baek SY, Sa SJ, Jeong YD, Cho ES, Hong JG, Kim YS, et al. Altrenogest affects expression of galectin-3 and fibroblast growth factor 9 in the reproductive tract of sows. Anim Biotechnol. 2020. https://doi.org/10.1080/10495398.2020.1726362

10. Chen X, Overstreet E, Wood SA, Fischer JA. On the conservation of function of the Drosophila fat facets deubiquitinating enzyme and Fam, its mouse homolog. Dev Genes Evol. 2000;210:603-10. https://doi.org/10.1007/s004270000109

11. Fischer-Vize JA, Rubin GM, Lehmann R. The fat facets gene is required for Drosophila eye and embryo development. Development. 1992;116:985-1000. https://doi.org/10.1242/ dev.116.4.985

12. Jones MH, Furlong RA, Burkin H, Chalmers IJ, Brown GM, Khwaja O, et al. The Drosophila developmental gene fat facets has a human homologue in Xp11.4 which escapes X-inactivation and has related sequences on Yq11.2. Hum Mol Genet. 1996;5:1695-701. https://doi. org/10.1093/hmg/5.11.1695

13. Brown GM, Furlong RA, Sargent CA, Erickson RP, Longepied G, Mitchell M, et al. Characterisation of the coding sequence and fine mapping of the human DFFRY gene and comparative expression analysis and mapping to the Sxrb interval of the mouse $\mathrm{Y}$ chromosome of the Dffry gene. Hum Mol Genet. 1998;7:97-107. https://doi.org/10.1093/hmg/7.1.97

14. Hall NM, Brown GM, Furlong RA, Sargent CA, Mitchell M, Rocha D, et al. Usp9y (ubiquitin-specific protease 9 gene on the $\mathrm{Y}$ ) is associated with a functional promoter and encodes an intact open reading frame homologous to Usp9x that is under selective constraint. Mamm Genome. 2003;14:437-47.https://doi.org/10.1007/s00335-002-3068-4

15. Taya S, Yamamoto T, Kanai-Azuma M, Wood SA, Kaibuchi K. The deubiquitinating enzyme Fam interacts with and stabilizes $\beta$-catenin. Genes Cells. 1999;4:757-67. https://doi. org/10.1046/j.1365-2443.1999.00297.x

16. Allrich RD, Christenson RK, Ford JJ, Zimmerman DR. Pubertal development of the boar: age-related changes in testicular morphology and in vitro production of testosterone and estradiol-17 $\beta$. Biol Reprod. 1983;28:902-9. https://doi.org/10.1095/biolreprod28.4.902

17. Ford JJ, Wise TH. Sertoli cell differentiation in pubertal boars. J Anim Sci. 2009;87:2536-43. https://doi.org/10.2527/jas.2009-1906 